\title{
Nasal carriage of Methicillin Resistant Staphylococcus aureus among health care workers in a tertiary care hospital
}

\author{
Sharanya K. ${ }^{1}$, Lakshmi K. ${ }^{2}$, Vinod K. ${ }^{3}$, Chitralekha Saikumar ${ }^{4}$ \\ ${ }^{1}$ Dr. Sharanya Krishnakumar, Assistant Professor, ${ }^{2}$ Dr. Lakshmi Krishnasamy, Associate Professor, ${ }^{3}$ Dr. Vinod Krishnagopal, \\ Professor, Department of Anaesthesiology, ${ }^{4}$ Dr. Chitralekha Saikumar, Professor and Head, ${ }^{1,2,4}$ authors are affiliated with \\ Department of Microbiology, All authors are affiliated with Sree Balaji Medical College \& Hospital (Bharath Institute of Higher \\ Education and Research), Chennai, Tamil Nadu, India.
}

Corresponding Author: Dr. Sharanya Krishnakumar, Assistant Professor of Microbiology, Sree Balaji Medical College and Hospital (BIHER), Chennai, Tamil Nadu, India. Email: sharanyavinod87@gmail.com

\begin{abstract}
Introduction: Hospital acquired infections especially with methicillin resistant Staphylococcus aureus (MRSA) is an important cause of morbidity and mortality. Staphylococcus aureus remains as a significant and potential pathogen because of its severity and increased frequency in causing a wide array of infections. The most common infections caused by $S$. aureus include skin and soft tissue, musculoskeletal system, respiratory system, central nervous system, endovascular system \&genitourinary system. It has become one of the leading causes of hospital acquired infections and are very difficult to treat because of increase in resistance to antimicrobial drugs. Aim and objectives: This study aims to screen for nasal carriage of Staphylococcus species, Methicillin Resistant Staphylococcus aureus (MRSA) and Methicillin Sensitive Staphylococcus aureus (MSSA) among the health care workers as they are potentially endangered target groups in a health care setting. Materials and Methods: The study was carried out in a tertiary care hospital for a period of 6 months from June 2018 to December 2018. Nasal swab samples were obtained from a total of 120 health care workers. Culture and sensitivity was performed by standard microbiological methods. Methicillin resistance was tested by using Cefoxitin disc $(30 \mu \mathrm{g})$ by Kirby-Bauer disk diffusion method on Mueller Hinton Agar. Results: Out of the 120 samples, 86 (72\%) samples were bacterial culture positive and 34(28\%) samples were culture negative. Among the 86 clinical isolates, 38 (31.6\%) were of Staphylococcus aureus, 26 (21.6\%) were of Coagulase negative Staphylococcus aureus (CONS) and 22 (18.3\%) samples were of bacteria other than Staphylococci. Conclusion: A higher rate of nasal carriage of MRSA was observed among the health care workers. Henceforth, routine screening of MRSA among the hospital staffs is recommended.
\end{abstract}

Keywords: Methicillin resistant Staphylococcus aureus, Nasal carriage, Health care workers.

\section{Introduction}

Staphylococcus is a facultative anaerobe appearing as Gram positive cocci in clusters when examined under light microscopy [1]. Large proportion of healthy population harbour and carry Staphylococcus aureus and Coagulase negative Staphylococcus (CONS) as normal commensal flora or as colonizer of various body sites especially the nose, skin surfaces etc. During favorable conditions Staphylococcal infection spreads by direct contact or indirectly by toxin production especially in health care settings [2]. This could be a reason for increased incidence and rapid spread of staphylococcal infections and (or) diseases where the original reservoir(s) of infection still remains unclear [3]. The situation appears still worse in the hospitals [4].

Manuscript received: $20^{\text {th }}$ October 2019 Reviewed: 30 30 $^{\text {th }}$ October 2019

Author Corrected: $5^{\text {th }}$ November 2019

Accepted for Publication: 11 $1^{\text {th }}$ November 2019
Penicillin resistance and the new $\beta$-lactamase resistant antibiotics like methicillin and oxacillin was found immediately after their clinical use in 1940s and 1960s respectively [5]; That is how methicillin resistant $S$. aureus [6] got its name. Anterior nares are considered as a main reservoir of Staphylococcus aureus both in adults and in children. Today the major nosocomial pathogen worldwide is Methicillin-resistant Staphylococcus aureus (MRSA). The surveillance of hospitals among various regions globally indicate the incidence of MRSA strains to be variable and individualized based on the hospital and the country involved [7]. Few hospitals recorded as high as $80 \%$ resistance [8].

This study aims to screen for nasal carriage of Staphylococcus species, Methicillin Resistant Staphylococcus aureus (MRSA) and Methicillin Sensitive

Pathology Update: Tropical Journal of Pathology \& Microbiology Available online at: www.medresearch.in 878 | P a g e 
Staphylococcus aureus (MSSA) among the health care workers as they are potentially endangered target groups in a health care setting ${ }^{1}$.

To also identify the presence of other species of Staphylococci especially coagulase negative Staphylococcus, viz., Methicillin Resistant Coagulase negative Staphylococci (MRCONS) and Methicillin Sensitive Coagulase negative Staphylococci (MSCONS).

\section{Materials and Methods}

Setting: This study was conducted among the nursing staff \& laboratory technicians of a tertiary care hospital at Chennai, Tamil Nadu in the Central laboratory.

Duration: June 2018 to December 2018

Type of study: Prospective cross-sectional study

Study subjects: A total of 120 subjects comprising 60 nursing staff \& 60 laboratory technicians were included in the study.

Ethical permission: The study was conducted after obtaining approval from Institutional Research and ethical committee.

Inclusion Criteria: Persons who are working as nursing staff or laboratory technician in the current institution for more than 6 months were included in the study.

Exclusion criteria: Persons with upper respiratory tract infections and who are currently on antibiotics and who underwent antibiotic therapy in the past 2 weeks were excluded from the study.

Procedure: After obtaining informed consent from the subjects, two nasal swabs were collected under aseptic precautions with sterile, dry cotton swabs from the anterior nares of each nostril about $1 \mathrm{~cm}$ depth and gently rotating the swab thrice.

They were then processed immediately or within 2 hours. The nasal swabs were inoculated in 5\% Sheep Blood Agar plate (BAP), Mac Conkey`s agar plate (MAC), Nutrient agar (NA)plates and were incubated at $37^{\circ} \mathrm{C}$ overnight.
Direct Gram Stain was performed for all nasal swabs and results noted. The plates were looked for the colony morphology and type of hemolysis in BAP, lactose fermentation in Mac Conkey's agar plate, golden yellow pigments in Nutrient agar plate.

If there was no growth after 24 hrs further incubation for a period of 48 hrs kept and declared as no growth. Slide catalase and tube coagulase tests were performed for suspected Staphylococcal colonies.

Biochemical reactions like Mannitol fermentation and Modified Hugh Leifson test (OF Test) were performed.

The Antibiotic Susceptibility Testing (AST) was performed on Mueller Hinton Agar (Hi Media Laboratories, Mumbai/ India) according to the CLSI with bacterial suspension of 0.5 Mac Farland turbidity standard.

The following antibiotics were tested by Kirby-Bauer disk

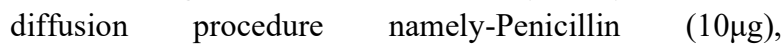
Erythromycin $(15 \mu \mathrm{g})$, Ciprofloxacin $(5 \mu \mathrm{g})$, Co-trimoxazole $(25 \mu \mathrm{g})$, Amikacin $(30 \mu \mathrm{g})$, Clindamycin $(2 \mu \mathrm{g})$, Cefoxitin $(30 \mu \mathrm{g}), \quad$ Linezolid $(30 \mu \mathrm{g}), \quad$ Teicoplanin $(30 \mu \mathrm{g}) \quad \&$ Gentamycin $(10 \mu \mathrm{g})$, Tetracycline $(30 \mu \mathrm{g})$.

Control strain: Staphylococcus aureus ATCC 25923 was used as reference standard control strain for every batch of culture and susceptibility testing.

Screening for Methicillin Resistance: Methicillin resistance was tested by using Cefoxitin disc $(30 \mu \mathrm{g})$ by Kirby-Bauer disk diffusion method on Mueller Hinton Agar.

Antibiotic Susceptibility Test patterns were read and subsequent identification of MRSA, MSSA, MRCONS, MSCONS were noted.

The zone of inhibition of $S$. aureus $\leq 21 \mathrm{~mm}$ and Coagulase negative Staphylococci $\leq 24 \mathrm{~mm}$ were considered as methicillin resistant.

Statistical analysis: The data obtained from the study were entered and analysed using SPSS windows version 14.0 software.

\section{Results}

A total of 120 health care workers were included in the study. Out of the 120 samples, 86 (72\%) samples were culture positive and $34(28 \%)$ samples were culture negative or sterile.

Among the 86 clinical isolates, 38 (31.6\%) were of Staphylococcus aureus, 26 (21.6\%) were of Coagulase negative Staphylococcus aureus and $22(18.3 \%)$ samples were of bacteria other than Staphylococci which is represented in the Figure 1 below.

Pathology Update: Tropical Journal of Pathology \& Microbiology Available online at: www.medresearch.in 879 | P a g e 


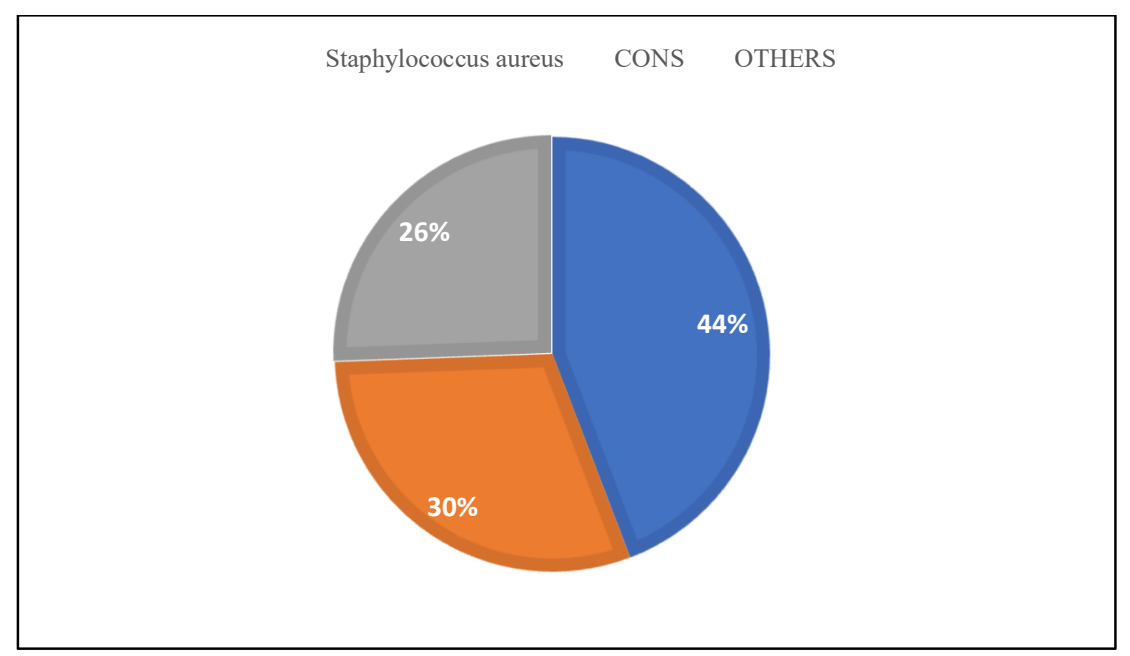

Fig-1: Culture positive isolates

Table-1: Characterization of staphylococcus species.

\begin{tabular}{|c|c|c|}
\hline Staphylococcal isolates & Number of isolates & Percentage of isolates \\
\hline MRSA & 30 & 25 \\
\hline MSSA & 8 & 6.6 \\
\hline MRCONS & 14 & 12 \\
\hline MSCONS & 12 & 10 \\
\hline
\end{tabular}

Table 1: MRSA- Methicillin resistant Staphylococcus aureus, MSSA - Methicillin Sensitive Staphylococcus aureus, MRCONS

- Methicillin resistant Coagulase Negative Staphylococcus, MSCONS - Methicillin Sensitive Coagulase Negative Staphylococcus

\section{Discussion}

Among the Gram positive organisms, S.aureus is one of the important pathogens causing skin and soft tissue infections. MRSA is found to be a major pathogen causing morbidity and mortality globally [9]. MRSA is found to be associated with increase in mortality and cost of health care systems [10].

Methicillin resistance arises following the acquisition of novel DNA, which results in production of a new penicillinbinding protein (PBP), known as PBP2' or PBP2a, which has low binding affinity for methicillin and other currently available $\beta$-lactams. Intermediate vancomycin or glycopeptide resistant Staphylococcus aureus were first detected in Japan in 1997[11], and subsequently in other countries [12,13]. In June 2002, the first Staphylococcus aureus isolate fully resistant to vancomycin (VRSA) was isolated in the USA [14]. Despite intensive attempts at eradication in the last two decades, MRSA continues to be the major nosocomial pathogen worldwide [15]. The level of hospital MRSA infection is indicative of the overall infection rate of the institution and usually reflects higher concentrations of patients, overcrowding of wards and heavy nursing load [5].
One of the important mode of transmission of MRSA within the hospitals is from patient-to-patient via the hands of hospital health care workers. Infected and colonized patients are the major reservoirs of the hospital MRSA. Colonization of hospital patients depends on the duration of hospital stay, nutritional status of the patient, recurrent or recent antibiotic treatment, presence of wound and/or invasive devices.

There is a high patient risk involved with surgical wound infection caused by hospital-acquired MRSA. Currently, most MRSA are hospital acquired and so this organism is a useful indicator of the effects of infection control per se. MRSA do not generally appear to be more virulent than sensitive strains but, because of their resistance patterns, they are more difficult to treat if infection occurs $[16,17]$.

Increase in number of MRSA among inpatients and occurrence of possible vancomycin resistance may indicate a need for quicker as well as reliable mode of identification and stop the spread of MRSA in hospitals. About 35-50\% of normal adults harbor Staphylococcus aureus at any instant [18]. 
The MRSA prevalence is found to be variable among the various countries ranging from very less $(0.4 \%)$ in Sweden to higher prevalence of $48.4 \%$ in Belgium [19]. In India the Prevalence of MRSA is found to be between $30 \%$ to $70 \%$ $[20,21]$. In another study from Chennai, Gopalakrishnan et al in 2010 found the prevalence of MRSA to be $40-50 \%$ [22].

Health care workers (HCWs) lie in between doctors and the patients. They border between hospitals and the community. The survey and investigations for MRSA carriage among the HCWs is mainly to look for any outbreaks or endemic MRSA nasal colonization. The MRSA colonization may predispose the HCWs to endogenous infections and further outcomes.

Many studies are being carried out globally to study the MRSA carriage rate among the HCWs. Studies reported that nasal carriage of MRSA strains varies between $18.2 \%$ to $43.8 \%$ among the HCWs $[23,24]$. However, to the best of our knowledge, the data regarding the epidemiology of MRSA among HCWs is still not sufficient. Transmission of infections between HCWs and from HCWs to patients is a major concern.

In the present study, subjects included 120 health care workers comprised of 60 nursing staffs and 60 lab technicians. In this study, the prevalence of S.aureus was $31.6 \%$ and MRSA was found to be $25 \%$ among the health care workers. The present results are similar with a recent study by Aila et al in 2017, where the nasal carriage of S.aureus was $31 \%$ and MRSA was $25.5 \%$ in their study [25]. Similar S.aureus colonization results have been reported in various other studies by Askarian et al [26] (31\%), Zorgani et al [27] (39\%), Kampf et al [28] (33.8\%), Elie-Turenne MC et al [23] (30\%). Goyal et al from Delhi, reported a higher prevalence of $50 \%$ S.aureus colonization among the HCWs [29]. The prevalence of MRSA in the present study was found to be higher than that reported in various other studies [30-32].

Antimicrobial resistance has become an emerging lifethreatening medical concern worldwide. Antibiotic resistance among the S.aureus is another wide spreading alarming issue to the treating physicians [33,34]. The MRSA strains are believed to be resistant to many classes of antimicrobial agents like aminoglycosides, macrolides, beta lactam antibiotics.

As a result, the treatment of MRSA infections has become difficult. The nasal carriage of MRSA among the HCWs increases the chance of spread of the organism among the vulnerable patients during the health care. The spread of MRSA can have harmful effects during outbreaks in resource limited settings where the microbiological investigations for bacterial identification and antibiotic susceptibility testing is difficult to be carried out. Large multicentric studies on MRSA nasal carriage is recommended.

Limitations of the study: The present study had limitations like smaller sample size. Further, Molecular methods like PCR based methods to detect MRSA carriers had not been carried out due to higher cost.

\section{Conclusion}

Staphylococcal species commonly reside as normal commensal flora of the nose of healthy individuals. Prevalence of MRSA in healthy individuals and evolving drug resistance could be potential target for translational research in the future.

Molecular methods to detect mecA gene of MRSA is a promising research tool to confirm the bacterial identification \& resistance pattern. Worldwide surveillance regimens are being introduced in hospitals to control MRSA infections. Success in the implementation of the infection control measures need good education, cooperation of the staffs and support from the hospital management.

Health care professionals need to be aware of the sanitary practices and antibacterial guidelines in order to prevent the spread of nosocomial infections. Routine handwashing practices, wearing personal protective equipment while examining the patients can decrease the risk of transmission of infectious diseases considerably. Periodical education and training should be given to the health care workers on the importance of health and infection control.

\section{What the study adds to the existing knowledge?}

The present study throws light in the rising trend in MRSA among the health care professionals and highlights the need for effective intervention and management.

\section{Author's contributions}

Dr. Sharanya Krishnakumar: Study concept, data collection, manuscript writing, final review and approval.

Dr. Lakshmi Krishnasamy, Dr. Vinod Krishnagopal: Manuscript writing, manuscript editing and review.

Dr. Chitralekha Saikumar: Study design and final approval

Funding: No funding sources

Conflict of interest: None declared

Ethical Approval: This study was approved by the Institutional Ethics Committee. 


\section{References}

1. Ranjani VP, Someshwaran R, Anbu N, Aravazhi A. Study on nasal carriage of staphylococcus species among medical and paramedical students in a tertiary care hospital, Coimbatore. Int J Curr Res. 2016;8(8):36365-36369.

2. Cosgrove SE, Sakoulas G, PerencevichEn, Scwaber MJ, Karchmer AW, Carmeli Y. Comparison of mortality associated with methicillin resistant and methicillin susceptible Staphylococcus aureus bacteremia: a metaanalysis. Clinical Infect Dis. 2003;36(1):53-59. doi:10. $1086 / 345476$

3. Christian Cespedes, Maureen Miller, Bianca Quagliarello, Peter Vavagiakis, Robert S. Klein, and Franklin D. Lowy. Differences between Staphylococcus aureus isolates from medical and Nonmedical Hospital Personnel. J Clinic Microbiol. 2002; 40(7): 2594-2597. doi: 10.1128/JCM.40.7.2594-2597.2002.

4. Sah P, Raj Rijal K, Shakya B Raj, B.T. Ghimire P. Nasal carriage rate of Staphylococcus aureus in hospital personnel of National Medical College and Teaching Hospital and their Antibiotic Susceptibility Pattern. JHAS. 2013;3(1):2123. doi: 10.12691/ajmr-2-4-1.

5. Lowy FD. Antimicrobial resistance: the example of Staphylococcus aureus. J Clin Invest. 2003; 111(9):12651273. doi:10.1172/JCI18535.

6. Ghidey F, Igbinosa O, Igbinosa E, Nasal colonization of methicillin resistant Staphylococcus aureus (MRSA) does not predict subsequent infection in the intensive care unit. $\mathrm{J}$ Basic App Sci. 2014;3(2):81-86. doi: https://doi.org/10. 1016/j.bjbas.2014. 03.001.

7. Sisirak M, Zvizdic A, Hukic M. Methicillin-resistant staphylococcus aureus (mrsa) as a cause of nosocomial wound infections. Bosnian J Basic Med Sci. 2010;10(1):3237. doi: $10.17305 /$ bjbms.2010.2733.

8. Campos M.L, Cipriano Z.M, Freitas P.F. Suitability of the NNIS index for estimating surgical-site infection risk at small university hospital in Brazil. Infect Control Hosp. Epidemiol.2001;22(5):268-272. doi:10.1086/501898.

9. Haddadin AH, Fappiano SA, Lipset PA. Methicillinresistant 4 (MRSA) in the intensive care unit. Postgrad Med J. 2002;78(921):385-392. doi:10.1136/pmj.78.921.385.

10. David MZ, Daum RS. Community-associated methicillin - resistant Staphylococcus aureus: epidemiology and clinical consequences of an emerging epidemic. Clin Microbiol Rev.2010;23(3):616-687. doi: 10.1128/CMR. 00081-09.
11. Hiramatasu K, Aritaka N, Hanaki H, Ino T, Yabuta K. Dissemination in Japanese hospitals of strains of Staphylococcus aureus heterogeneously resistant to vancomycin. Lancet 1997:350,1670-1673. doi:10.1016/ S0140-6736 (97)07324-8.

12. Tenover F.C, Lancaster M.V, Hill B.C. Characterization of staphylococci with reduced susceptibilities to vancomycin and other glycopeptides. J Clin Microbiol. 1998; 36(7):1020-1027.

13. Kim M.N, Pai C.H, Woo Ryu J.H, Hiramatsu K. Vancomycin intermediate Staphylococcus aureus in Korea. J Clin Microbiol.2000;38(10):3879-3881.

14. Srinivasan A, Dick J.D, Perl T.M. Vancomycin resistance in staphylococci. Clin Microbiol Rev. 2002;(15) : 430-438.doi:10.1128/cmr.15.3.430-438.2002.

15. National Nosocomial Infections Surveillance System. National Nosocomial Infections Surveillance (NNIS) System Report, data summary from January 1992 [ through June 2004] issued October 2004. Am J Infect Control.2004; 32(8):470-485. doi:10.1016/S0196655304005425.

16. Kalenic S. The Resistance of Bacteria to Antibiotics. Medicus.2009;9(2):149-158.

17. Damani N.N. Methicillin-resistant Staphylococcus aureus. Manual of Infection Control Procedures. 2003; 121-129.

18. Bannerman Tl,Murray PR, Baron EJ, Jorgensen JH, Pfaller MA, Yolken RH. Staphylococcus, Micrococcus and other catalase-positive cocci that grow aerobically In: Manual of clinical microbiology 8. Th. Washington. DC. ASM Pres.2003:383-404.

19. Sader HS, Farrell DJ, Jones RN. Antimicrobial susceptibility of Gram-positive cocci isolated from skin and skin-structure infections in European medical centres. Int J Antimicrob Agents. 2010; 36:28-32. doi: 10.1016/j. ijantimicag. 2010.03.016.

20. Verma S, Joshi S, Chitnis V, Hemwani N, Chitnis D. Growing problem of methicillin resistant Staphylococci: Indian scenario. Indian J Med Sci. 2000; 54(12): 535540 .

21. Rajaduraipandi K, Mani KR, Panneerselvam K, Mani M, Manikandan P. Prevalence and antimicrobial susceptibility pattern of methicillin resistant S. aureus: a multicentre study. Indian J Med Microbiol.2006;26(1):3438. doi:10.4103/0255-0857.19892. 
22. Gopalakrishnan R, Sureshkumar D. Changing trends in antimicrobial susceptibility and hospital acquired infections over an 8 year period in a tertiary care hospital in relation to introduction of an infection control programme. J Assoc Physicians India. 2010;58:25-31.

23. Elie-Turenne MC, Fernandes H, Mediavilla JR, Rosenthal M, Mathema B, Singh A, et al. Prevalence and characteristics of Staphylococcus aureus colonization among healthcare professionals in an urban teaching hospital. Infect Control Hosp Epidemiol.2010;31(6):574580. doi: 10.1086/652525.

24. Akhtar N. Staphylococcal nasal carriage of health care workers. J Coll Phys Surg.2010;20(7):439-443. doi: 07. 2010 / JCPSP.439443.

25. El Aila NA, Al Laham NA, Ayesh BM. Nasal carriage of methicillin resistant Staphylococcus aureus among health care workers at Al Shifa hospital in Gaza Strip. BMC Infect Dis. 2017;17:28. doi:10.1186/s12879-016-2139-1.

26. Askarian M, Zeinalzadeh A, Japoni A, Alborzi A, Memish ZA. Prevalence of nasal carriage of methicillinresistant Staphylococcus aureus and its antibiotic susceptibility pattern in healthcare workers at Namazi Hospital, Shiraz, Iran. Int J Infect Dis. 2009;13(5):e241247. doi:10.1016/j.ijid.2008.11.026.

27. Zorgani A, Elahmer O, Franka E, Grera A, Abudher A, Ghenghesh KS. Detection of meticillin-resistant Staphylococcus aureus among healthcare workers in Libyan hospitals. J Hosp Infect. 2009; 73(1):91-92. doi:10. 1016/ j.jhin. 2009.06.019.

28. Kampf G, Adena S, Ruden H, Weist K. Inducibility and potential role of MecA-gene-positive oxacillin-susceptible
Staphylococcus aureus from colonized healthcare workers as a source for nosocomial infections. J Hosp Infect. 2003; 54 (2): 124-129. doi:10.1016/s0195-6701(03)00119-1.

29. Goyal R, Das S, Mathur M. Colonisation of methicillin resistant Staphylococcus aureus among health care workers in a tertiary care hospital of Delhi. Indian J Med Sci. 2002;56(7):321-324.

30. Malini J, Shruti AH, Padmavathy M, Umapathy BL, Navaneeth BV, KeerthiMannan J, Girish MS. Methicillinresistant Staphylococcus aureus carriage among the health care workers in a tertiary care hospital. JCDR. 2012; 6(5): 791-793.

31. Khanal R, Sah P, Lamichhane P, Lamsal A, Upadhaya S, Pahwa VK. Nasal carriage of methicillin resistant Staphylococcus aureus among health care workers at a tertiary care hospital in Western Nepal. Antimicrob Resist Infect Control. 2015; 4:39. doi: 10.1186/s13756-0150082-3

32. Shibabaw A, Abebe T, Mihret A. Nasal carriage rate of methicillin resistant Staphylococcus aureusamongdessie referral hospital health care workers; dessie, northeast Ethiopia. Antimicrob Resist Infect Control. 2013;2(1):25. doi: 10.1186/2047-2994-2-25.

33. Stefani S, Chung DR, Lindsay JA, et al. Methicillin resistant Staphylococcus aureus (MRSA): global epidemiology and harmonization of typing methods. Int $\mathrm{J}$ Antimicrob Agents.2012; 39(4):273-282. doi: 10.1016/j. ijantimicag. 2011.09.030.

34. Appelbaum PC. Microbiology of antibiotic resistance in Staphylococcus aureus. Clin Infect Dis. 2007;45(3):171176. doi:10.1086/519474.

\section{How to cite this article?}

Sharanya K, Lakshmi K, Vinod K, Chitralekha Saikumar. Nasal carriage of Methicillin Resistant Staphylococcus aureus among health care workers in a tertiary care hospital. Trop J Path Micro 2019;5(11):878-883.doi:10.17511/jopm.2019.i11.08 